\title{
Heat Transfer in Inclined Enclosure of Silica Aerogel/Glass Fiber Composite Material
}

\author{
Manal H. Saleh \\ Department of Mechanical \\ Engineering \\ University of Baghdad
}

\author{
Amina H. Dhaef \\ Department of Mechanical \\ Engineering \\ University of Wasit
}

\begin{abstract}
An investigation is performed for natural convection of air in a three dimensional inclined annulus enclosure. The annulus material is made of silica aerogel/glass fiber composite materials. The annulus enclosure is filled with silica sand between two inclined concentric cylinders with fins attached to the inner cylinder. Constant walls temperature boundary conditions are considered under steady state condition. The parameters affected on the system are modified Rayleigh number $\left(10 \leq \mathrm{Ra}^{*} \leq 500\right)$ and the annulus inclination angle $\delta$ $\left(0^{\circ}, 30^{\circ}, 45^{\circ}, 60^{\circ}\right.$ and $\left.90^{\circ}\right)$. The effect of fibres filler in composite material is investigated and two values of effective thermal conductivity are performed: the minimum and the maximum values considering the fiber alignment effect. The results showed insignificant effect of the inclination angle on the average Nusselt number for the low values of $\mathrm{Ra}^{*}$. The average $\mathrm{Nu}$ number increases with an increase in modified Rayleigh number and decrease with the increase of $\delta$ for high values of $\mathrm{Ra}^{*}$. The deviation between the average $\mathrm{Nu}$ for the maximization and minimization of the thermal conductivity is equal to $2.26 \%$ for $\delta=90^{\circ}$ (horizontal annulus) and $0.46 \%$ for $\delta=0^{\circ}$ (vertical annulus). A correlation for the average Nusselt number in terms of $\mathrm{Ra}^{*}$ and $\delta$, has been developed for the outer cold cylinder and for the two cases of maximization and minimization of thermal conductivity.
\end{abstract}

\section{General Terms}

$\mathrm{Cp}$ : Specific heat at constant pressure $\left(\mathrm{kJ} / \mathrm{kg}{ }^{\circ} \mathrm{C}\right)$, g: Acceleration due to gravity $\left(\mathrm{m} / \mathrm{s}^{2}\right), \quad H_{f}$ : Fin length $(\mathrm{m}), H_{l}$ : Dimensionless fin length $\left(\begin{array}{lll}H_{f} & r_{\text {out }}\end{array}\right), k_{\text {fin }}$ : Fin thermal conductivity (W/m K), $\mathrm{k}_{\mathrm{f}}$ : Thermal conductivity of the fluid $(\mathrm{W} / \mathrm{m} \mathrm{K}), \mathrm{k}_{\mathrm{eff}}$ : Effective thermal conductivity of the porous media (W/m K), K: Permeability $\left(\mathrm{m}^{2}\right)$, 1: Cylinder length (m), L:Dimensionless cylinder length, Nu1: Local Nusselt number on the inner cylinder, Nu2: Local Nusselt number on the outer cylinder, $\mathrm{Nu}_{\mathrm{in}}$ : Average Nusselt number on the inner cylinder, $\mathrm{Nu}_{\text {out }}$ : Average Nusselt number on the outer cylinder p: Pressure $(\mathrm{N} / \mathrm{m} 2), q$ Local heat flux $\left(\mathrm{W} / \mathrm{m}^{2}\right), r_{i n}$ : Radius of the inner cylinder $(\mathrm{m}), r_{\text {out }}$ : Radius of the outer cylinder $(\mathrm{m})$, $\mathrm{R}$ : Dimensionless radial coordinate, Ra*: Modified Rayleigh number, $R r$ : radius ratio, $S$ : Fin pitch $(\mathrm{m}), S_{I}$ and $S_{2}$ : Dimensionless fin spacing, T: Temperature $(\mathrm{K}), \mathrm{u}, v$ and $\mathrm{w}$ : velocity components in $\mathrm{r}, \phi$ and $\mathrm{z}$ - direction $(\mathrm{m} / \mathrm{s}), \mathrm{U}, \mathrm{V}, \mathrm{W}$ : Dimensionless velocity component in $\mathrm{R}, \phi$ and $\mathrm{Z}$ direction, $\mathrm{x}$, y, z: Cartesian coordinate system (m), Z: Dimensionless axial coordinate, $\alpha_{e f f}$ : Effective thermal diffusivity $\left(\mathrm{m}^{2} / \mathrm{s}\right), \alpha_{m}$ : Medium thermal diffusivity $\left(\mathrm{m}^{2} / \mathrm{s}\right), \beta$ : Volumetric thermal expansion coefficient $(1 / / \mathrm{K}), \delta$ : Angle of inclination (degree), $\theta$ : Dimensionless temperature,

\section{Keywords}

Natural convection, inclined annulus, laminar flow, silica sand, silica aerogel/glass fiber.

\section{INTRODUCTION}

In many applications a material of high corrosion resistance, high strength-to-density ratio, stiffness-to-density ratio, and plasticity is needed and the fiber reinforced multilayer composite materials is found to be very suitable for these applications as compared with most materials. Two properties cause the advantages of these materials, which are the fibers orientations in every layer; and having different physical, mechanical and thermal properties of various materials to meet the design requirements. The applications of these materials are in fluid reservoirs, pipes pressure vessels and aerospace and marine industries and it used in various designs to improve the characteristic of various constructions and reduce their weight. One of the factor that influences the resultant value of a property of a composite material (which is made of two or more materials) as a whole is its geometrical structure. The properties of these materials are commonly called effective properties of a composite. [1] Examined the effect of multi fibres filler in composite materials on thermal conductivity. To find the optimal arrangement of fibres position in composite matrix, three types of optimization were performed in terms of effective thermal conductivity. Hybrid method combining optimization with genetic algorithm and differential equation solver by finite element method were used. [2] Presented a theoretical and experimental study on thermal conductivities of several silica aerogel composite insulation materials. The results show that the density of the silica is the key factor affecting the effective thermal conductivity of these composite insulation materials, and the density of aerogel has little influence. [3] Fabricated silica aerogel/glass fiber composites by press forming of silica aerogel powders and dispersed glass fibers. Due to the nano porous structure, silica aerogel showed low gas thermal conductivity and solid thermal conductivity. By adding the glass fibers, the strength of the composites was improved but the heat insulation property was deteriorated. The thermal conductivities of the composites with $20 \mathrm{wt} . \%$ glass fibers at $300{ }^{\circ} \mathrm{C}$ and $600{ }^{\circ} \mathrm{C}$ were $0.025 \mathrm{~W} /(\mathrm{m} \mathrm{K})$ and $0.030 \mathrm{~W} /(\mathrm{m} \mathrm{K})$, respectively. The addition of $\mathrm{TiO} 2$ decreased the radiative thermal conductivity abruptly, especially at high temperatures. At $700{ }^{\circ} \mathrm{C}$, it decreased from $0.041 \mathrm{~W} /(\mathrm{m} \mathrm{K})$ to $0.030 \mathrm{~W} /(\mathrm{m}$ $\mathrm{K})$ after $20 \mathrm{wt} . \% \mathrm{TiO} 2$ powders were added. [4] investigate the radiative properties and heat transfer in fiber-loaded silica aerogel composites using modified theory in a combined heat conduction and radiation model. To simulate a very realistic material structure, a randomly parameterized 2-D fiber distribution was generated. The finite volume method was used to solve a two flux radiation model and the steady-state energy equation to calculate the effective thermal conductivity of the composite. Theoretic guidelines for material designs with optimum parameters were obtained from the numerical results, such as the inclination angle, diameter and length-todiameter ratio of the fibers. It was found that the fiber extinction coefficient increases as the fiber length-to-diameter ratio is reduced or the fiber inclination angle is increased. 
Reducing the fiber length-to diameter ratio and the inclination angle and increasing the fiber volume fraction cause the effective thermal conductivity of the fiber-loaded aerogel to be reduced. [5]By considering the heat conduction and thermal radiation simultaneously a complete computing procedure for calculating the thermal conductivities of silica aerogel composite insulating materials was obtained. A proposed of a sphere model was taken for the nano-porous silica aerogel in which the scale effect on gas conduction and solid-matrix conduction were both considered. Mie theory used to determine the radiative properties of additives (opacifier particles and fibers), which are needed for calculating the radiative conductivity in Rossland equation. The results show that the total conductivity decreases first and then increases as the mass fraction of additive increases. [6] Investigated the thermal radiative transfer in silica aerogel and silica aerogel composite insulation materials. To determine the specific spectral extinction coefficient and the specific Rosseland mean extinction coefficient of each sample, the spectral transmittances were used. The results show that the spectral extinction coefficients of the samples are strongly dependent on the wavelength, particularly in the short wavelength regime. Increasing the temperature cause to decrease the total Rosseland means extinction coefficients of all the samples. As sample densities increase, the radiative conductivities which are almost proportional to the cube of the temperature decreased.

In the present research the natural convection heat transfer is investigated in an inclined annulus enclosure used as heat exchanger. The material taken for the annulus was based on previous experimental work which used silica aerogel/glass fiber composite material. The ranges of the parameters affected on the study are modified Rayleigh number $\left(10 \leq \mathrm{Ra}^{*} \leq 500\right)$ and the annulus inclination angle $\delta(0,30,45$, 60 and 90). Two values of effective thermal conductivity will be performed as the minimum and the maximum values depending on the fiber alignment where the Silica aerogel/glass fiber composite material has a thermal conductivity in parallel direction of fibers equal $0.15 \mathrm{~W} / \mathrm{m} \mathrm{K}$ and in perpendicular direction of fibers of $0.08 \mathrm{~W} / \mathrm{m} \mathrm{K}$ [5].

\section{MATHEMATICAL MODEL All}

material on each page should fit within a rectangle of $18 \mathrm{x}$ $23.5 \mathrm{~cm}\left(7 " \mathrm{x} 9.25^{\prime \prime}\right)$, centered on the page, beginning $2.54 \mathrm{~cm}$ (1") from the top of the page and ending with $2.54 \mathrm{~cm}(1 ")$ from the bottom. The right and left margins should be $1.9 \mathrm{~cm}$ (.75"). The text should be in two $8.45 \mathrm{~cm}(3.33 ")$ columns with a $.83 \mathrm{~cm}(.33 ")$ gutter. The schematic drawing of the geometry and the Cartesian coordinate system employed in solving the problem is shown in Fig.1. The steady-state equations of the Darcy flow model, which are, the mass, the momentum (Darcy), the energy conservation laws and the Boussinesq's approximation are applied. [7] Gave the vectorial notation of these equations.

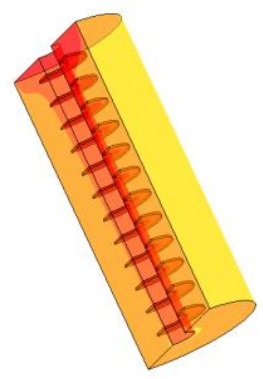

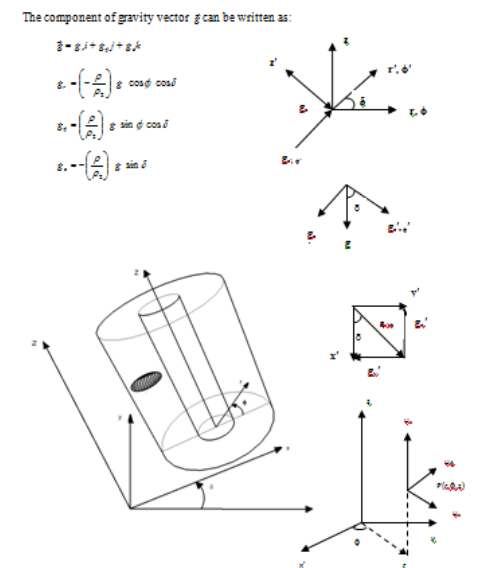

Fig.1 Coordinates system and geometry

\section{GOVERNING EQUATIONS}

The conservation equations of mass, momentum and energy in steady state and the supplementary equation are as follow:

$\rho=\rho_{\text {in }}\left\{1-\beta\left(T-T_{\text {in }}\right)\right\}$

Where:

$\beta=\frac{1}{\rho} \frac{\partial \rho}{\partial T}$

$\beta$ Is the thermal coefficient of the volume expansion, this constant is evaluated at $T_{\text {in }}$ which is the temperature at the inner surface of the outer cylinder, $\rho_{\text {in }}$ is the density at $T_{\text {in }}$ and $\rho$ is the density at T, [8]. This technique is called Boussinesq's approximation.

\subsection{Mass Conservation}

$$
\frac{\partial u}{\partial r}+\frac{u}{r}+\frac{1}{r} \frac{\partial v}{\partial \phi}+\frac{\partial w}{\partial z}=0
$$

\subsection{Momentum Equations}

The volume average velocity through the porous material is proportional with the pressure gradient, so the Darcy's model in three dimensional flows [9] is:

\subsubsection{Momentum Equation in Radial Direction}

$u=\frac{K}{\mu_{f}}\left[-\frac{\partial p}{\partial r}-\rho g \cos \phi \cos \delta\right]$

\subsubsection{Momentum Equation in Angular Direction}

$v=\frac{K}{\mu_{f}}\left[-\frac{1}{r} \frac{\partial p}{\partial \phi}+\rho g \sin \phi \cos \delta\right]$

\subsubsection{Momentum Equation in Axial Direction}

$w=\frac{K}{\mu_{f}}\left[-\frac{\partial p}{\partial z}-\rho g \sin \delta\right]$

\subsubsection{Energy Equation}

$$
\begin{aligned}
& \frac{\partial\left(\rho C_{p} T\right)}{\partial t}+u \frac{\partial}{\partial r}\left(\rho C_{p} T\right)+\frac{v}{r} \frac{\partial}{\partial \phi}\left(\rho C_{p} T\right)+w \frac{\partial}{\partial z}\left(\rho C_{p} T\right)= \\
& \frac{1}{r} \frac{\partial}{\partial r}\left\{r \frac{\partial(k T)}{\partial r}\right\}+\frac{1}{r^{2}} \frac{\partial^{2}(k T)}{\partial \phi^{2}}+\frac{\partial^{2}(k T)}{\partial z^{2}}+\mu \Phi
\end{aligned}
$$

Where $\Phi$ is viscous dissipation function 


\subsubsection{Fin Equation}

The energy equation of fins is given by [10]. Twelve fins are attached to the inner cylinder and the equation is as follow:

$\frac{\partial T}{\partial r}+\frac{T}{r}+\frac{1}{r} \frac{\partial T}{\partial \theta}+\frac{\partial T}{\partial z}=0$

A vorticity vector $\Omega$ and a vector potential $\Psi$ with its components is as [11]:

$\left.\Psi=\left(\psi_{r}, \psi_{\phi}, \psi_{z}\right)\right)$

Defined by:

$\bar{U}=\alpha_{\text {eff. }} \nabla \times \Psi$

$\nabla^{2} \psi_{r}=\frac{1}{R} \frac{\partial W}{\partial \phi}-\frac{\partial V}{\partial Z}$

$\nabla^{2} \psi_{\phi}=\frac{\partial U}{\partial Z}-\frac{\partial W}{\partial R}$

$\nabla^{2} \psi_{Z}=\frac{1}{R} \frac{\partial(R V)}{\partial R}-\frac{1}{R} \frac{\partial U}{\partial \phi}$

\section{NON DIMENSIONAL VARIABLES}

For the present study, the characteristic length is taken as $r_{\text {out }}$ and the dimensionless magnitudes are defined as follow:

$R=\frac{r}{r_{\text {out }}}, \quad Z=\frac{z}{r_{\text {out }}}, \quad U=\frac{u l}{\alpha_{m}}, \quad V=\frac{v l}{\alpha_{\text {eff }}}, \quad W=\frac{w l}{\alpha_{\text {eff }} .}$,

$\theta=\left(T-T_{\text {out }}\right) /\left(T_{\text {in }}-T_{\text {out }}\right)$,

$$
P=\frac{p K l}{\alpha_{\text {eff }} \mu_{f} r_{\text {out }}}
$$

$R a^{*}=g \beta K\left(T_{\text {in }}-T_{\text {out }}\right)\left(r_{\text {out }}-r_{\text {in }}\right) / \alpha_{\text {eff. }} v$

$S_{1}=\frac{s}{2 r_{\text {out }}} S_{2}=\frac{\frac{s}{2}+t}{r_{\text {out }}} \quad H_{1}=\frac{H_{f}}{r_{\text {out }}}$

Substitute these dimensionless magnitudes in the governing equations. Alternative expressions of eq. (3) may be written in terms of $\psi_{r}, \psi_{\phi}$ and $\psi_{z}$ as :

$U=\left(\frac{1}{R} \frac{\partial \psi_{z}}{\partial \phi}-\frac{\partial \psi_{\phi}}{\partial Z}\right)$

$V=\left(\frac{\partial \psi_{r}}{\partial Z}-\frac{\partial \psi_{z}}{\partial R}\right)$

$W=\frac{1}{R}\left\{\frac{\partial}{\partial R}\left(R \psi_{\phi}\right)-\frac{\partial \psi_{r}}{\partial \phi}\right\}$

To eliminate pressure terms, take curl of momentum equations and it will be:

$R a^{*} \frac{l}{\left(r_{\text {out }}-r_{\text {in }}\right)}\left(\frac{1}{R} \sin \delta \frac{\partial \theta}{\partial \phi}+\sin \phi \cos \delta \frac{\partial \theta}{\partial Z}\right)=$

$-\frac{\partial^{2} \psi_{r}}{\partial R^{2}}-\frac{1}{R^{2}} \frac{\partial\left(R \psi_{r}\right)}{\partial R}-\frac{2}{R} \frac{\partial \psi_{r}}{\partial R}-\frac{1}{R^{2}} \frac{\partial^{2} \psi_{r}}{\partial \phi^{2}}-\frac{\partial^{2} \psi_{r}}{\partial Z^{2}}-\frac{2}{R} \frac{\partial \psi_{z}}{\partial Z}$

(16)

$$
\begin{aligned}
& R a^{*} \frac{l}{\left(r_{\text {out }}-r_{\text {in }}\right)}\left(\cos \phi \cos \delta \frac{\partial \theta}{\partial Z}-\sin \delta \frac{\partial \theta}{\partial R}\right)= \\
& -\frac{\partial^{2} \psi_{\phi}}{\partial Z^{2}}-\frac{\partial^{2} \psi_{\phi}}{\partial R^{2}}-\frac{1}{R^{2}} \frac{\partial^{2} \psi_{\phi}}{\partial \phi^{2}}-\frac{2}{R^{2}} \frac{\partial \psi_{r}}{\partial \phi}+\frac{\psi_{\phi}}{R^{2}}-\frac{1}{R} \frac{\partial \psi_{\phi}}{\partial R} \\
& -R a^{*} \frac{l}{\left(r_{\text {out }}-r_{\text {in }}\right)} \cos \delta\left(\frac{1}{R} \cos \phi \frac{\partial \theta}{\partial \phi}+\sin \phi \frac{\partial \theta}{\partial R}\right)= \\
& -\frac{\partial^{2} \psi_{z}}{\partial R^{2}}-\frac{1}{R} \frac{\partial \psi_{z}}{\partial R}-\frac{1}{R^{2}} \frac{\partial^{2} \psi_{z}}{\partial \phi^{2}}-\frac{\partial^{2} \psi_{z}}{\partial Z^{2}}
\end{aligned}
$$

The vector potential equation was obtained in the dimensionless form as

$$
\begin{aligned}
& \nabla^{2} \psi_{r}=-\frac{\partial^{2} \psi_{r}}{\partial R^{2}}-\frac{1}{R^{2}} \frac{\partial\left(R \psi_{r}\right)}{\partial R}-\frac{2}{R} \frac{\partial \psi_{r}}{\partial R}-\frac{1}{R^{2}} \frac{\partial^{2} \psi_{r}}{\partial \phi^{2}}-\frac{\partial^{2} \psi_{r}}{\partial Z^{2}}-\frac{2}{R} \frac{\partial \psi_{z}}{\partial Z} \\
& \nabla^{2} \psi_{\phi}=-\frac{\partial^{2} \psi_{\phi}}{\partial Z^{2}}-\frac{\partial^{2} \psi_{\phi}}{\partial R^{2}}-\frac{1}{R^{2}} \frac{\partial^{2} \psi_{\phi}}{\partial \phi^{2}}-\frac{2}{R^{2}} \frac{\partial \psi_{r}}{\partial \phi}+\frac{\psi_{\phi}}{R^{2}}-\frac{1}{R} \frac{\partial \psi_{\phi}}{\partial R} \\
& \nabla^{2} \psi_{z}=-\frac{\partial^{2} \psi_{z}}{\partial R^{2}}-\frac{1}{R} \frac{\partial \psi_{z}}{\partial R}-\frac{1}{R^{2}} \frac{\partial^{2} \psi_{z}}{\partial \phi^{2}}-\frac{\partial^{2} \psi_{z}}{\partial Z^{2}}
\end{aligned}
$$

And the energy equation will be:

$$
\begin{aligned}
& \left(\frac{1}{R} \frac{\partial \psi_{z}}{\partial \phi}-\frac{\partial \psi_{\phi}}{\partial Z}\right) \frac{\partial \theta}{\partial R}+\frac{1}{R}\left(\frac{\partial \psi_{r}}{\partial Z}-\frac{\partial \psi_{z}}{\partial R}\right) \frac{\partial \theta}{\partial \phi} \\
& +\left(\frac{\psi_{\phi}}{R}+\frac{\partial \psi_{\phi}}{\partial R}-\frac{1}{R} \frac{\partial \psi_{r}}{\partial \phi}\right) \frac{\partial \theta}{\partial Z} \\
& =\frac{l}{r_{i n}}\left[\frac{\partial^{2} \theta}{\partial R^{2}}+\frac{1}{R} \frac{\partial \theta}{\partial R}+\frac{1}{R^{2}} \frac{\partial^{2} \theta}{\partial \phi^{2}}+\frac{\partial^{2} \theta}{\partial Z^{2}}\right]
\end{aligned}
$$

And fin equation will be:

$\frac{\partial \theta}{\partial R}+\frac{\theta}{R}+\frac{1}{R} \frac{\partial \theta}{\partial \phi}+\frac{\partial \theta}{\partial Z}=0$

\subsection{Dimensionless Hydraulic Boundary \\ Conditions}

For the vector potential field, the boundary conditions are given as:

$$
\begin{array}{lr}
\frac{1}{R} \frac{\partial}{\partial R}\left(R \psi_{r}\right)=\psi_{\phi}=\psi_{z}=0 & \text { at } R=R_{1}, 1 \\
\psi_{r}=\frac{\partial \psi_{\phi}}{\partial \phi}=\psi_{z}=0 & \text { at } \phi=0, \pi \\
\psi_{r}=\psi_{\phi}=\frac{\partial \psi_{z}}{\partial Z}=0 & \text { at } Z=0, L
\end{array}
$$

And for the fin, the boundary conditions are given as:

$\frac{1}{R} \frac{\partial}{\partial R}\left(R \psi_{r}\right)=\frac{\partial \psi_{\phi}}{\partial \phi}=\frac{\partial \psi_{Z}}{\partial Z}=0$

On the fin faces which were located on the following planes (fin base)

At $\mathrm{R}=R_{1} \quad$ for $\quad \phi=0, \pi$ 
As shown in Fig.2

(fin tip)

$$
\begin{aligned}
& \text { At } \quad r=r_{i n}+H_{f} \quad \text { for } \quad \phi=0, \pi \\
& \text { At } S_{1} \text { and } S_{2} \quad \text { for any } r \text { and } \phi
\end{aligned}
$$

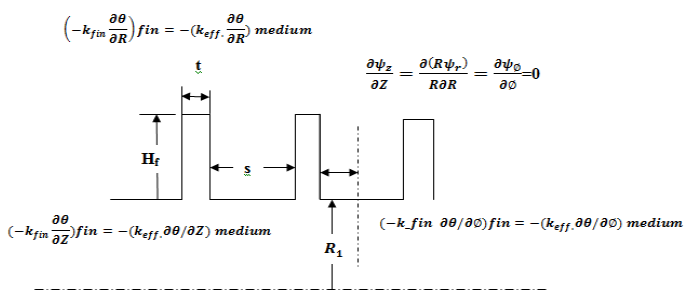

Fig. 2 fin boundary conditions

\subsection{Dimensionless Thermal Boundary Conditions}

For the temperature field, the dimensionless thermal boundary conditions are:

$$
\begin{aligned}
& \theta=1 \\
& \text { at } R=R_{1}=r_{\text {in }} / r_{\text {out }} \\
& \theta=0 \\
& \text { at } R=R_{2}=1 \\
& \frac{\partial \theta}{\partial \phi}=0 \\
& \text { at } \phi=0, \pi \\
& \frac{\partial \theta}{\partial Z}=0 \\
& \text { at } Z=0, L \\
& \text { at } R=H_{1} \\
& -\left.k_{f i n} \frac{\partial \theta}{\partial R}\right|_{\text {fin }}=-\left.k_{\text {eff. }} \cdot \frac{\partial \theta}{\partial R}\right|_{\text {medium }} \\
& \text { at } S_{1} \text { at any } R \text { and } \phi \\
& \text { at } S_{2} \quad \text { at any } R \text { and } \phi \\
& -\left.k_{\text {fin }} \frac{\partial \theta}{\partial Z}\right|_{\text {fin }}=-\left.k_{\text {eff. }} \frac{\partial \theta}{\partial Z}\right|_{\text {medium }} \\
& \text { at } \phi=0, \pi \quad \text { and } \text { any } R \quad-\left.k_{\text {fin }} \frac{\partial \theta}{\partial \phi}\right|_{f i n}=-\left.k_{\text {eff. }} \frac{\partial \theta}{\partial \phi}\right|_{\text {medium }} \\
& k_{e f f}=(1-\varepsilon) k_{s}+\varepsilon k_{f}
\end{aligned}
$$

The fiber orientation influences the conduction heat transfer. Conduction heat transfer would get the minimum value when fiber axis is perpendicular to heat flux, and conductive conductivity calculated by series model would be the lowest. Conductive conductivity calculated by parallel model would be the largest when fiber axis is parallel to heat flux. The thermal conductivity of the silica aerogel/glass fiber composite laminate parallel to the fibers is greater than that perpendicular to the fibers. Silica aerogel/glass fiber composite material has a thermal conductivity in parallel direction of fibers of $0.15 \mathrm{~W} / \mathrm{m} \mathrm{K}$ and in perpendicular direction of fibers of $0.08 \mathrm{~W} / \mathrm{m} \mathrm{K}$ [5].

\section{COMPUTATIONAL TECHNIQUE}

Equations (16, 17, 18, 22 and 23) were transformed into the finite difference equations, where the upwind differential method in the left hand side of the energy eq.(22) and the centered - space differential method for the other terms were used, and solved by using (SOR) method [9]. To obtain the results of the problem a computer program was built using MATLAB-7 program. The value of the vector potential $\psi$ will be calculated at each node, in which the value of vector potential is unknown, the other node will appear in the right hand side of each equation. As an initial value of iteration, zero is chosen for the vector potential field, while a conduction solution is adopted for temperature field. The index (n) was used to represent the nth - approximation of temperature denoted by $\theta^{n}$ and substituted into the approximated equations, which were solved to obtain the nth approximation of vector potential $\psi$, then $\psi$ was substituted into eq. (22) to obtain $\theta^{n+1}$. The procedure is repeated until reach a convergence which is given by as

$\operatorname{Max}\left|\frac{\theta^{n+1}-\theta^{n}}{\theta^{n}}\right| \leq 10^{-8}$

The number of grid points used was 21 grid points in the $\mathrm{R}$ direction, 31 in the $\phi$-direction and 301 in the $\mathrm{Z}$ - direction which seems reasonable.

\section{CALCULATION OF LOCAL AND AVERAGE NUSSELT NUMBER}

Local Nusselt number is the dimensionless parameter indicative of the rate of energy convection from a surface and can be obtained as follows [8]:

$N u=\frac{q\left(r_{\text {out }}-r_{\text {in }}\right)}{k\left(T_{\text {in }}-T_{\text {out }}\right)}$

The local Nusselt number $N u_{1}$ and $N u_{2}$ on the inner and the outer cylinders are written as [8]:

$$
\begin{aligned}
& N u_{1}=-\left(1-R_{1}\right)\left(\frac{\partial \theta}{\partial R}\right)_{R=R_{1}} \\
& N u_{2}=-\left(1-R_{1}\right)\left(\frac{\partial \theta}{\partial R}\right)_{R=1_{1}}
\end{aligned}
$$

The average Nusselt number $N u_{\text {in }}$ and $N u_{\text {out }}$ on the inner and the outer cylinders are defined as:

$$
\begin{aligned}
& N u_{\text {in }}=-\left(1-R_{1}\right) \frac{1}{\pi L} \int_{0}^{L} \int_{0}^{\pi}\left(\frac{\partial \theta}{\partial R}\right)_{R=R} d \phi d Z \\
& N u_{\text {out }}=-\left(1-R_{1}\right) \frac{1}{\pi L} \int_{0}^{L} \int_{0}^{\pi}\left(\frac{\partial \theta}{\partial R}\right)_{R=1} d \phi d Z
\end{aligned}
$$

\section{RESULTS AND DISCUSSION}

Fig.3 to Fig. 6 show the variation in the average Nusselt number on the outer cold cylinder with the inclination angle for different values of $\mathrm{Ra}^{*}$ and for the two cases of the minimum and maximum values of thermal conductivity respectively. It is clear that $\mathrm{Nu}$ decrease with the increase of $\delta$ where the zero degree inclination is for vertical cylinder and $90^{\circ}$ for horizontal cylinder. These figures also show that the average Nusselt number increase with the increase of $\mathrm{Ra}^{*}$. 


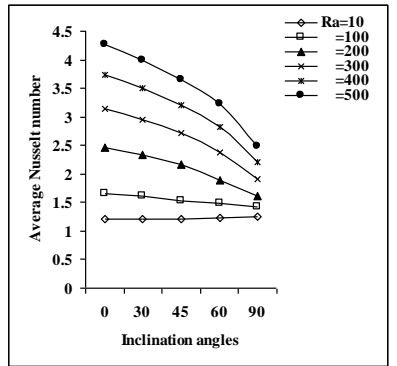

Fig.3 Variation of $\mathrm{Nu}_{\text {average }}$ with the inclination angle on the outer cold cylinder for $k=0.08 \mathrm{~W} / \mathrm{m} \mathrm{K}$

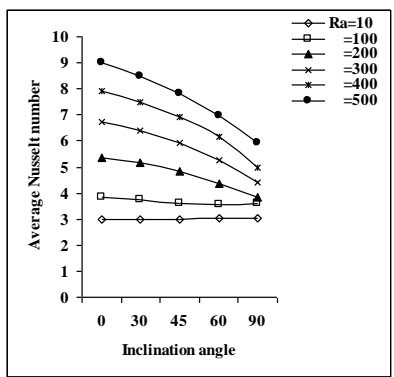

Fig.4 Variation of $\mathrm{Nu}_{\text {average }}$ with the inclination angle on the inner hot cylinder for $\mathrm{k}=0.08 \mathrm{~W} / \mathrm{m} \mathrm{K}$

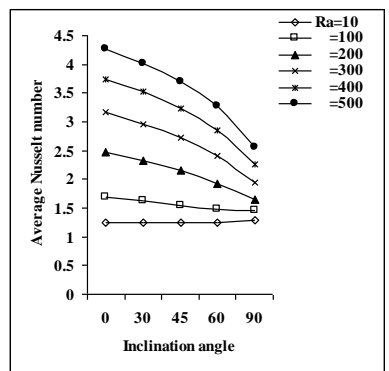

Fig.5 Variation of $\mathrm{Nu}_{\text {average }}$ with the inclination angle on the outer cold cylinder for $k=0.15 \mathrm{~W} / \mathrm{m} \mathrm{K}$

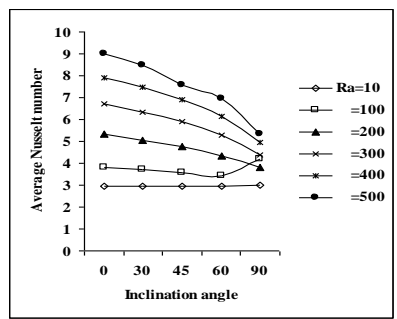

Fig.6 Variation of $\mathrm{Nu}_{\text {average }}$ with the inclination angle on the inner hot cylinder for $\mathrm{k}=0.15 \mathrm{~W} / \mathrm{m} \mathrm{K}$

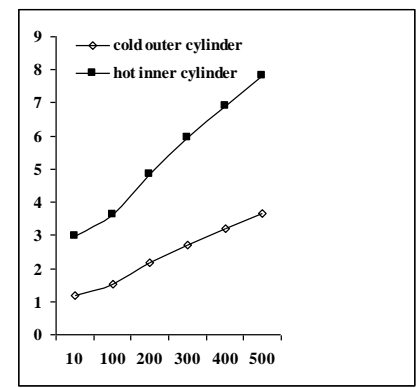

Fig. 7 Variation of $\mathrm{Nu}_{\text {average }}$ with $\mathrm{Ra}$ on the outer cold cylinder for $\delta=0^{\circ}$

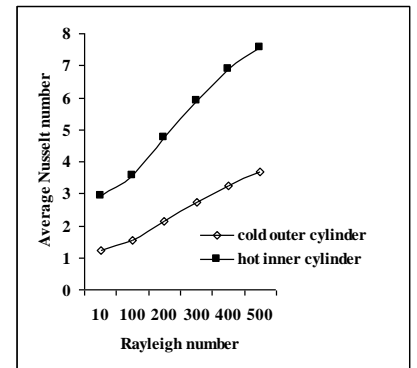

Fig. 8 Variation of $\mathrm{Nu}_{\text {average }}$ with $\mathrm{Ra}$ on the outer cold cylinder for $\delta=\mathbf{9 0}^{\circ}$

The deviation between the average $\mathrm{Nu}$ in the two cases is clear in Fig.7 and Fig. 10 which is equal to $2.26 \%$ for $\delta=90^{\circ}$ and $0.46 \%$ for $\delta=0^{\circ}$. For the inner hot cylinder the deviation between the two cases is not significant for $\delta=0^{\circ}$ but it will be significant only for vertical annulus at $\mathrm{Ra}^{*}=500$ and equal to $23.44 \%$.

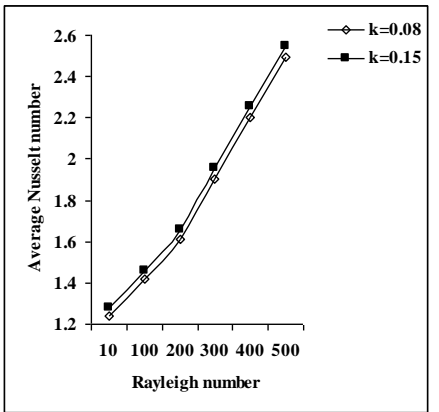

Fig. 9 Variation of $\mathrm{Nu}_{\text {average }}$ with $\mathrm{Ra}$ on the inner hot cylinder for $\delta=0^{\circ}$

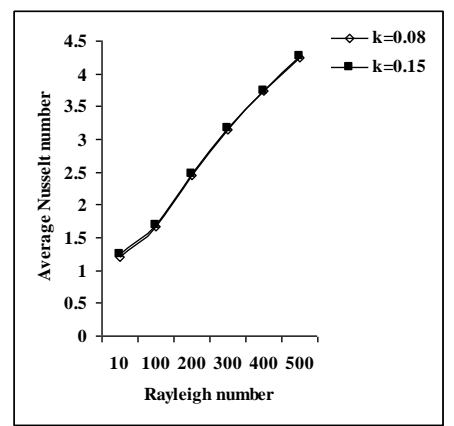

Fig. 10 Variation of $\mathrm{Nu}_{\text {average }}$ with $\mathrm{Ra}$ on the inner hot cylinder for $\delta=90^{\circ}$

Distribution of local Nusselt number along the circumstance of the cold and hot cylinders is illustrated in Fig. 11 to Fig. 26 respectively for the minimization and maximization of thermal conductivity and at three locations; located at the top of the cylinder, at the center of the cylinder and the third at the bottom of the cylinder. The local Nusselt number on the hot wall had a decreasing trend and there were three regions which could be distinguished. The first region, where the local Nusselt number approached to its maximum values this was due to the formation of plume in this region. Formation of the plume occurred where the two convective currents coming from the two annulus halves, impinging with each other and moving together upward without mixing, leaving a relatively stagnant region under impinging point. 


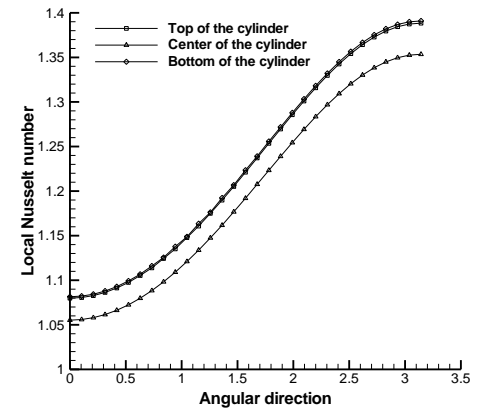

Fig. 11 Variation of $\mathrm{Nu}_{\text {Local }}$ in angular direction on the cold cylinder for $\mathbf{R a}=10 \mathrm{k}=\mathbf{0 . 0 8} \mathrm{W} / \mathrm{m} \mathrm{K} \delta=0^{\circ}$

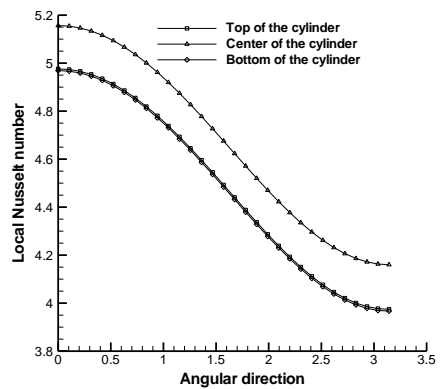

Fig. 12 Variation of $\mathrm{Nu}_{\text {Local }}$ in angular direction on the hot cylinder for $\mathrm{Ra}=10 \mathrm{k}=0.08 \mathrm{~W} / \mathrm{m} \mathrm{K} \delta=0^{\circ}$

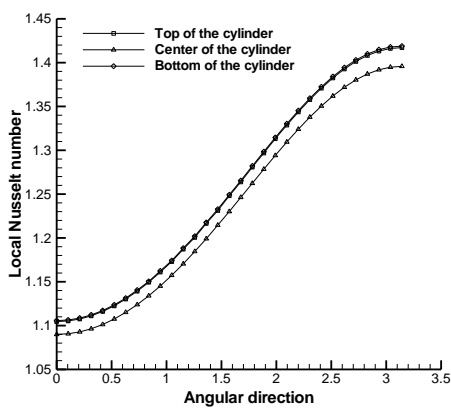

Fig. 13 Variation of $\mathrm{Nu}_{\mathrm{Local}}$ in angular direction on the cold cylinder for $\mathrm{Ra}=10 \mathrm{k}=\mathbf{0 . 1 5} \mathrm{W} / \mathrm{m} \mathrm{K} \delta=0^{\circ}$

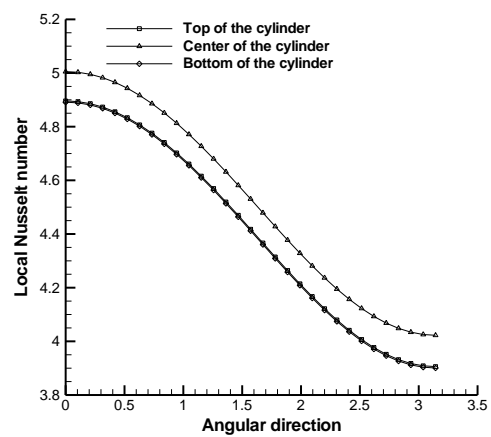

Fig. 14 Variation of $\mathrm{Nu}_{\text {Local }}$ in angular direction on the cold cylinder for $\mathrm{Ra}=10 \mathrm{k}=0.15 \mathrm{~W} / \mathrm{m} \mathrm{K} \delta=0^{\circ}$

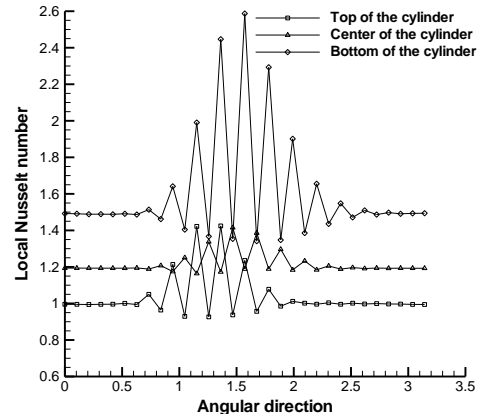

Fig. 15 Variation of $\mathrm{Nu}_{\text {Local }}$ in angular direction on the cold cylinder for $R a=10 \mathrm{k}=0.08 \mathrm{~W} / \mathrm{m} \mathrm{K} \delta=90^{\circ}$

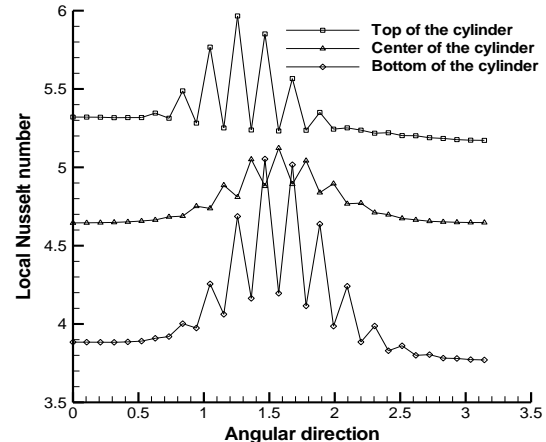

Fig. 16 Variation of $\mathrm{Nu}_{\text {Local }}$ in angular direction on the hot cylinder for $\mathrm{Ra}=10 \mathrm{k}=0.08 \mathrm{~W} / \mathrm{m} \mathrm{K} \delta=90^{\circ}$

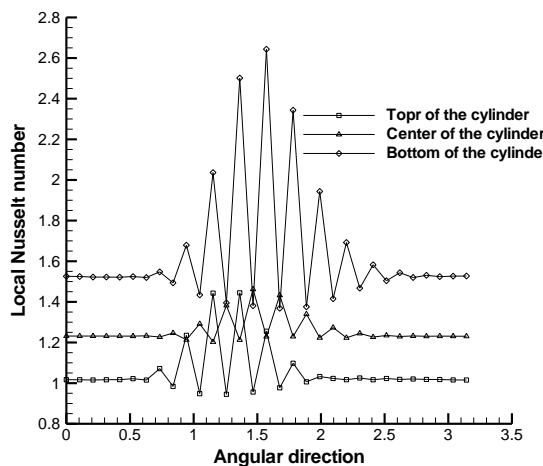

Fig. 17 Variation of $\mathrm{Nu}_{\text {Local }}$ in angular direction on the cold cylinder for $\mathrm{Ra}=10 \mathrm{k}=0.15 \mathrm{~W} / \mathrm{m} \mathrm{K} \delta=90^{\circ}$

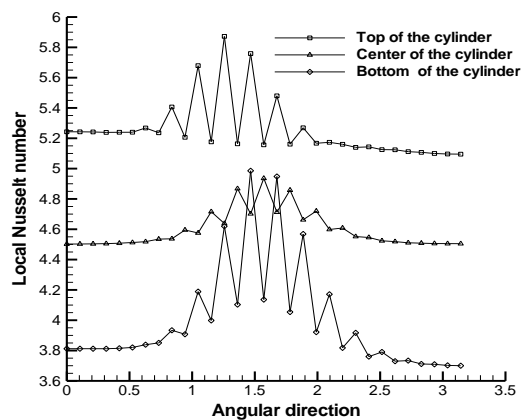

Fig. 18 Variation of $\mathrm{Nu}_{\text {Local }}$ in angular direction on the hot cylinder for $\mathrm{Ra}=10 \mathrm{k}=\mathbf{0 . 1 5} \mathrm{W} / \mathrm{m} \mathrm{K} \delta=90^{\circ}$ 


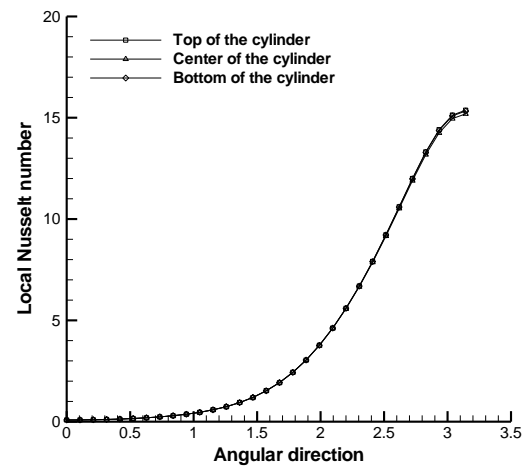

Fig. 19 Variation of $\mathrm{Nu}_{\text {Local }}$ in angular direction on the cold cylinder for $\mathrm{Ra}=500 \mathrm{k}=0.08 \mathrm{~W} / \mathrm{m} \mathrm{K} \delta=0^{\circ}$

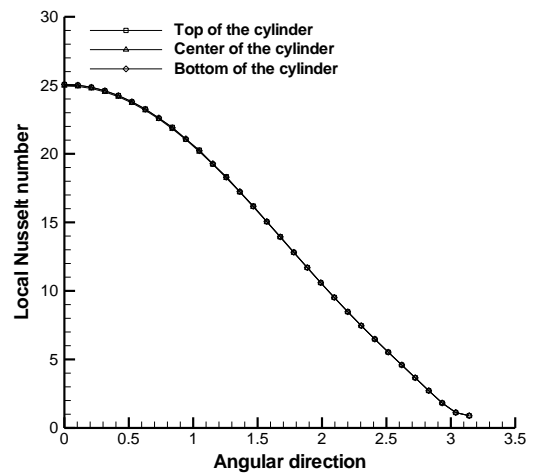

Fig. 20 Variation of $\mathrm{Nu}_{\text {Local }}$ in angular direction on the hot cylinder for $\mathbf{R a}=500 \mathrm{k}=0.08 \mathrm{~W} / \mathrm{m} \mathrm{K} \delta=0^{\circ}$

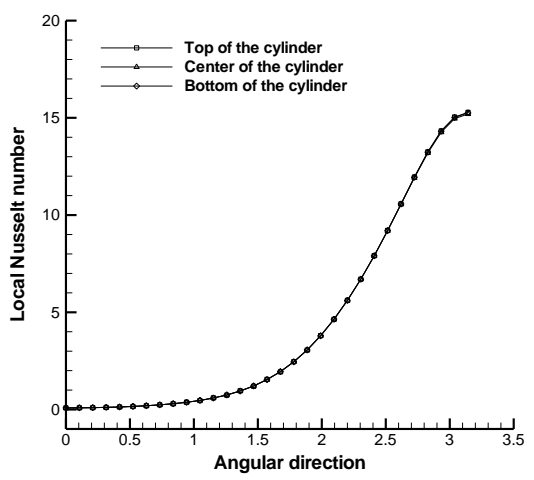

Fig. 21 Variation of $\mathrm{Nu}_{\mathrm{Local}}$ in angular direction on the cold cylinder for $\mathrm{Ra}=500 \mathrm{k}=0.15 \mathrm{~W} / \mathrm{m} \mathrm{K} \delta=0^{\circ}$

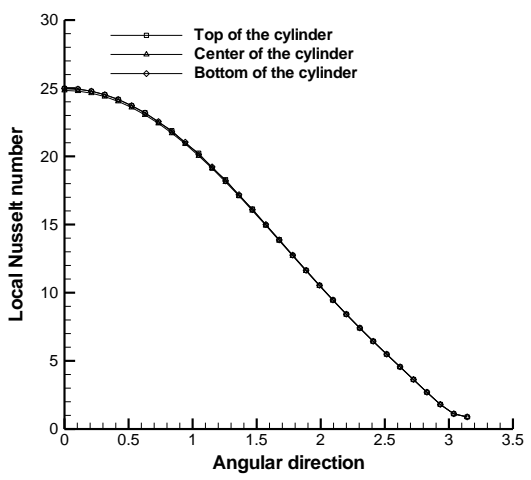

Fig. 22 Variation of $\mathrm{Nu}_{\text {Local }}$ in angular direction on the hot cylinder for $\mathbf{R a}=500 \mathrm{k}=0.15 \mathrm{~W} / \mathrm{m}$ K $\delta=0^{\circ}$

The second region, where the local Nusselt number loosed the uniformity and a large gradient in its distribution could be observed and this is because the inner boundary layer got thicker in this region since heat removal by fluid decrease as the fluid ascend up. The third region, where the local Nusselt number is relatively constant since the inner boundary layer of relative uniform thickness and give a little variation in local Nusselt number distribution. This behavior is roughly similar for most of modified Rayleigh number whereas the behavior of the cold wall is to increase. As shown in these figures, the maximum value of the local Nusselt number at horizontal position $\left(\delta=90^{\circ}\right)$ of the annulus. At high $\mathrm{Ra}^{*}$ in Fig. 19 to Fig. 26 the local Nusselt number was high owing to the effect of convection mode of heat transfer, at low $\mathrm{Ra}^{*}$ the mode of heat transfer is conduction and its value increase with the increase of $\mathrm{Ra}^{*}$ and it is clear that the second region will be vanish or in other words the variation trend to be uniform. Fig. 23 to Fig. 26 for $\delta=90^{\circ}$, there is only two regions and the non uniformity of the local Nusselt number will be significant.

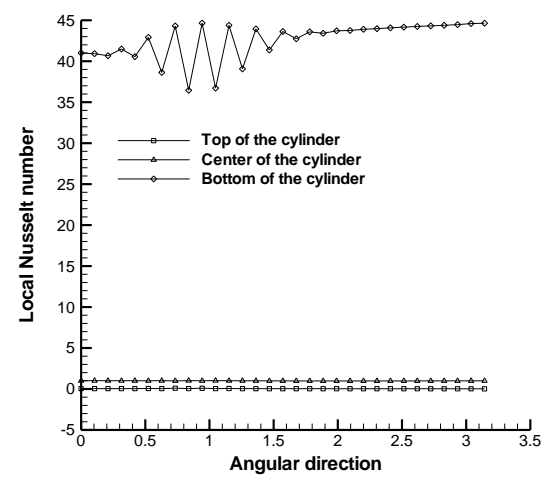

Fig. 23 Variation of $\mathrm{Nu}_{\text {Local }}$ in angular direction on the

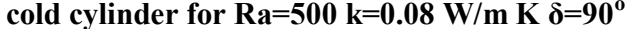




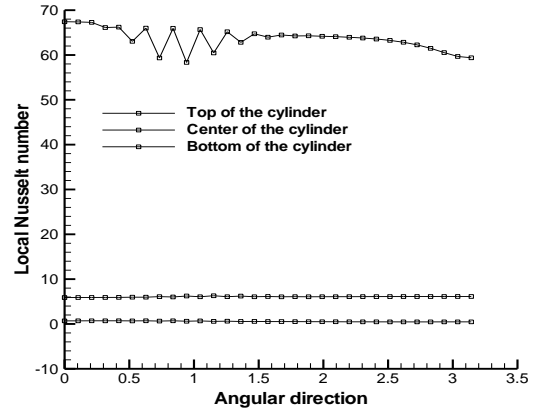

Fig. 24 Variation of $\mathrm{Nu}_{\text {Local }}$ in angular direction on the hot cylinder for $\mathrm{Ra}=500 \mathrm{k}=0.08 \mathrm{~W} / \mathrm{m} \mathrm{K} \delta^{\delta}=90^{\circ}$

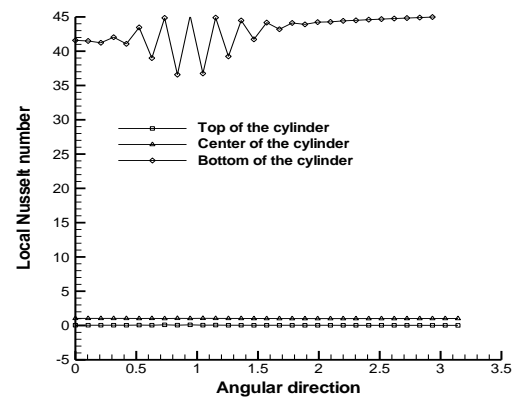

Fig. 25 Variation of $\mathrm{Nu}_{\text {Local }}$ in angular direction on the cold cylinder for $\mathrm{Ra}=500 \mathrm{k}=0.15 \mathrm{~W} / \mathrm{m} \mathrm{K} \delta=90^{\circ}$

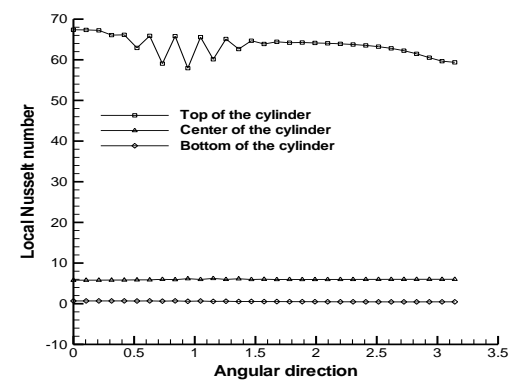

Fig. 26 Variation of $\mathrm{Nu}_{\text {Local }}$ in angular direction on the hot cylinder for $\mathrm{Ra}=500 \mathrm{k}=0.15 \mathrm{~W} / \mathrm{m} \mathrm{K} \delta^{\mathrm{\delta}}=\mathbf{9 0 ^ { \circ }}$

Correlations were deduced from the numerical data which are given as:

For k=0.15 W/m K:

$N u_{\text {out }}=\frac{0.685 R a^{0.239}}{\delta^{9.438}}$

For $\mathrm{k}=0.08 \mathrm{~W} / \mathrm{m} \mathrm{K}$ :

$N u_{\text {out }}=\frac{0.607 R a^{0.2585}}{\delta^{1.034}}$

\section{CONCLUSIONS}

The following conclusions can be obtained from the present study:

1- The deviation between the average $\mathrm{Nu}$ for the maximization and minimization of the thermal conductivity is equal to $2.26 \%$ for $\delta=90^{\circ}$ (horizontal annulus) and $0.46 \%$ for $\delta=0^{\circ}$ (vertical annulus).
2- Local $\mathrm{Nu}$ increases in the angular direction for the outer cold cylinder and has a trend to decrease on the inner hot cylinder.

3- Results showed that the average $\mathrm{Nu}$ number increases with an increase in modified Rayleigh number and decrease with the increase of $\delta$ for high values of $\mathrm{Ra}^{*}$, but hardly affected by $\delta$ for low values of $\mathrm{Ra}^{*}$.

4- It is recommended for future work to investigate the forced convection heat transfer in a pipe made of the same material (silica aerogel/glass fiber composite materials) and with different boundary conditions.

\section{REFERENCES}

[1] Hubert Jopek and Tomasz Strek, 2011, Optimization of the effective thermal conductivity of a composite, Convection and Conduction Heat Transfer, Poznan University of Technology, Institute Of Applied Mechanics Poland, 17, October, 197-214.

[2] Gaosheng Wei, Yusong Liu, Xinxin Zhang, Fan Yu, Xiaoze Du, 2011, Thermal conductivities study on silica aerogel and its composite insulation Materials, International Journal of Heat and Mass Transfer 54, 2355-2366.

[3] Bo Yuan, Shuqiang Ding, Dongdong Wang, Gang Wang, Hongxia Li, 2012, Heat insulation properties of silica aerogel/glass fiber composites fabricated by press forming, Materials Letters 75, 204-206.

[4] Jun-Jie Zhao, Yuan-Yuan Duan, Xiao-Dong Wang, BuXuan Wang, 2012, Radiative properties and heat transfer characteristics of fiber-loaded silica aerogel composites for thermal insulation, International Journal of Heat and Mass Transfer 55, 5196-5204

[5] Tao Xie, Ya-Ling He, Zi-Jun Hub, 2013, Theoretical study on thermal conductivities of silica aerogel composite insulating material, International Journal of Heat and Mass Transfer 58, 540-552

[6] Gaosheng Wei, Yusong Liu, Xinxin Zhang, Xiaoze Du, 2013, Radiative heat transfer study on silica aerogel and its composite insulation materials, Journal of NonCrystalline Solids 362, 231-236.

[7] Nield D. A. and Bejan A., "Convection in Porous Media”, Springer-Verlag, New York, 1999.

[8] Fukuda K., Takata Y., Hasegawa S., Shimomura H. and Sanokawa K., 1980, Three - Dimensional Natural Convection in a Porous Medium Between Concentric Inclined Cylinders, Proc. $19^{\text {th }}$ Natl Heat Transfer Conf., Vol. HTD -8, $97-103$.

[9] Wang Bu - Xuan and Zhang Xing, 1990, Natural Convection in Liquid Saturated Porous Media Between Concentric Inclined Cylinders, Int. J. Heat and Mass Transfer Vol. 33. No 5, 827-833.

[10] Ramón L. F. and Sergio G. M., 2007, Three Dimensional Natural Convection in Finned Cubical Enclosure, Int. J. of Heat and Fluid Flow. Vol. 28,289-298.

[11] Aziz K. and Hellums J. D., 1967, Numerical Solution of the Three Dimensional Equations of Motion for Laminar Natural Convection, The Physics of Fluids, V01. 10, No. $2,314-324$. 